\title{
Preference for Randomization: Empirical and Experimental Evidence
}

\author{
Nadja Dwenger* \\ Dorothea Kübler** \\ Georg Weizsäcker***
}

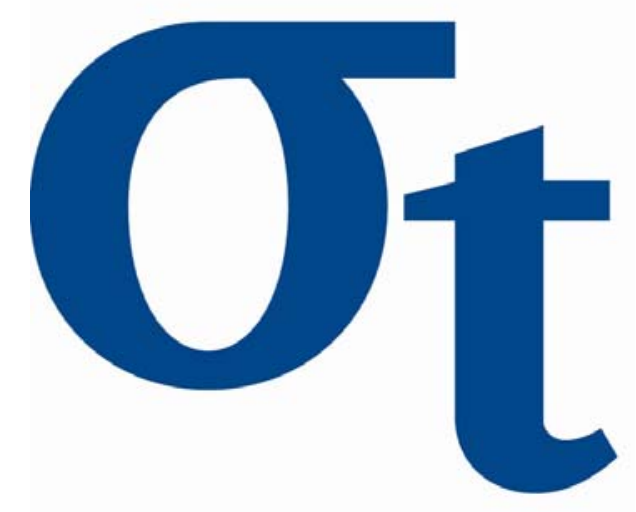

9)

$\forall$

6

* Max Planck Institute for Tax Law and Public Finance Munich, Germany

** Social Science Research Centre Berlin (WZB)

$\&$ Technical University Berlin, Germany

*** Humboldt Universität zu Berlin \& German Institute of Economic Research (DIW Berlin) \& University College London

This research was supported by the Deutsche Forschungsgemeinschaft through the SFB 649 "Economic Risk".

$$
\begin{gathered}
\text { http://sfb649. wiwi. hu-berlin. de } \\
\text { ISSN 1860-5664 }
\end{gathered}
$$




\title{
Preference for Randomization: Empirical and Experimental Evidence ${ }^{1}$
}

\author{
Nadja Dwenger \\ Max Planck Institute for Tax Law and Public Finance Munich \\ and \\ Dorothea Kübler \\ Social Science Research Centre Berlin (WZB) \\ \& Technical University Berlin \\ and \\ Georg Weizsäcker ${ }^{2}$ \\ Humboldt Universität Berlin \\ \& German Institute of Economic Research (DIW Berlin) \\ \& University College London
}

\footnotetext{
${ }^{1}$ We thank the ZVS - particularly Bernhard Scheer, Harald Canzler and Uwe Kuhnen-for providing us with the university admission data set and for additional information. We are grateful for helpful comments by Eddie Dekel, Frank Heinemann, and audiences at Birmingham, Innsbruck, MPI Jena, TU Berlin, UCL, Zurich and D-TEA 2010, Paris. Financial support through CRC 649 ("Economic Risk") is gratefully acknowledged

${ }^{2}$ Contact: nadja.dwenger@tax.mpg.de, kuebler@wzb.eu, weizsaecker@hu-berlin.de
} 


\title{
Preference for Randomization: Empirical and Experimental Evidence
}

\begin{abstract}
We investigate violations of consequentialism in the form of the stochastic dominance property. The property is shared by many theories of choice and implies that the decision-maker prefers receiving the best outcome for sure over all lotteries that involve multiple outcomes. We run experiments to demonstrate that dominated randomization can be attractive. In treatments where decision-makers are asked to submit multiple decisions without knowing which one is relevant, many participants submit contradictory sets of decisions and thereby induce a dominated lottery between outcomes. Explicit choice of non-consequentialist randomization is observed in a separate treatment. A possible reason for the effect is the desire to avoid having to make the decision. A large data set on (high-stake) university applications in Germany shows patterns that are consistent with a preference for randomization.
\end{abstract}

Keywords: Stochastic dominance violations, individual decision making, university choice, matching

JEL Classification: D03; D01 


\section{Introduction}

In situations where decision-making is hard, a possible procedural preference arises: the decision-maker may wish for the decision to be taken away from herself. Her cognitive or emotional cost of deciding may outweigh the benefits that arise from making the optimal choice. For example, the decision-maker may prefer not to make a choice without having sufficient time and energy to think it through. Or, she may not feel entitled to make it. Or, she may anticipate a possible disappointment about her choice that can arise after a subsequent resolution of uncertainty. Or, she may perceive the revelation of a particular preference embarrassing vis-à-vis others. Waiving some or all of the decision right seems desirable in such circumstances even though it typically increases the chance of receiving a suboptimal outcome.

The difficulty of such preferences is that they are non-consequentialist and are therefore excluded by most models of choice such as expected utility. For example, flipping a coin between different choice options contradicts expected utility theory except if the decisionmaker is exactly indifferent between these options. More general than expected utility theory, a basic axiom of choice - stochastic dominance - postulates that whenever the decision-maker has a strict preference for one of the options, she makes the choice herself rather than delegate it to randomness.

This paper discusses several data sets, experimental and field-empirical, where stochastic dominance is violated by decision makers. The largest data set concerns an important decision in people's lives: university applications. When applying to the German clearinghouse for university admission in one of the centrally administered fields of study, the (fairly complicated) quota rules require each applicant to submit multiple rank-order lists of universities knowing that each of the lists could be relevant to determine her university seat allocation. Our main empirical observation is that between the multiple submitted rank-order lists, uni- 
versity applicants often contradict themselves. Thereby, they effectively delegate the outcome to an uncertain process that is suboptimal under standard assumptions. Had an applicant always reported the same rank-order list instead of producing a preference reversal between her submitted lists, she would have reduced the probability of ending up at less desirable universities. In our cleanest (and most conservative) calculation, which excludes rational reasons for choice reversals (see Section 3.3.3), we find that $14 \%$ of applicants exhibit such behavior. Among these, we estimate that $17 \%$ are assigned to a university that they prefer less than a university to which they would have been assigned in the absence of a preference reversal.

Yet, in many economic contexts the decision-makers have good reasons for not making a decision. Especially due to informational reasons, it is often rational to use one of three avoidance strategies: delay, default, or delegation. For example, it may be important for the decision-maker to remain flexible if potential news can arrive in the future. Or, there may exist an exogenous agent or decision mechanism that use better information, making it optimal to leave the choice to them. These reasons are, however, unlikely to apply in the university choice data as the applicants know that their rank-order lists cannot be changed after the arrival of new information and that the mechanism selecting the lists does not utilize much choice-relevant information. Yet another possible rationalization of the preference reversals in university choice - one that we cannot rule out - are irrational beliefs of the decision-makers. The allocation mechanism of the central clearing house is highly complicated and depends on the submitted preferences of thousands of applicants $3^{3}$ Allowing for non-rational beliefs on behalf of the applicants, a large set of choice patterns can be optimal.

We therefore turn to a set of simple choice experiments, all conducted in large undergraduate lectures, that demonstrate a preference for randomization. In low-stake classroom

\footnotetext{
${ }^{3}$ The mechanism is analyzed in Braun et al. (2010) and Braun et al. (2012), pointing to a particular strategic feature of the mechanism: the fact that the mechanism involves a sequence of procedures with fixed assignment of seats creates dynamic incentive effects. These dynamic incentive effects strongly affect the truthtelling incentives at the early stages because the applicants have to consider the effect of moving to the next procedure. In this paper we use these results to rule out that strategic effects may lead to preference reversals that look like a preference for randomization.
} 
experiments, the importance of making the optimal choice is far lower than in the university admissions process. Indeed, we deliberately use decision problems where the typical participant is relatively close to indifferent as she is asked to choose between vouchers that have similar monetary values. But the experimental format allows us to observe a "pure" preference for randomization. The decision problems are so simple that informational reasons, including beliefs about the optimal choice, are implausible. Substantial proportions of participants (between $15 \%$ and $53 \%$ ) nevertheless choose a random outcome, violating stochastic dominance. In order to rule out that participants are exactly indifferent between the available voucherswhich would rationalize the pattern — we use control groups and separate preference elicitation questions that show that the participant population has a clear preference order over the set of available options. The responses to the control questions also show that random-utility models (akin to assuming that subjects are close to indifference) cannot explain the data patterns $4^{4}$ We therefore conclude that the observed choice of randomized outcomes, giving suboptimal outcomes a non-zero chance of being realized, appears to be deliberate.

Several of our experiments and the university-application process share the feature that the decision makers choose between the same options repeatedly. In these experiments, subjects choose twice, and a random draw determines which of the two choices is payoff relevant. The design thereby allows the participants to put option A ahead of option B in their first choice, and vice versa in their second choice. Indeed we observe that sizable numbers of participants (15-40\%, in different treatments) show such reversals. The simplest version of this is the binary-choice format of Experiment I (Section 2.1), where two options are available on each of two choice lists, and $28 \%$ of participants indicate a preference reversal between their two choices. In a control group that makes the choice between the options only once, we observe that the average choice leans more heavily in favor of one option.

\footnotetext{
${ }^{4} \mathrm{~A}$ sizable literature analyzes whether previously found biases in decision experiments may be generated by models of random choice. See e.g. Berg et al. (2010) for a recent study on preference reversals.
} 
The experimental evidence is not limited to choice reversals. A separate treatment (Experiment II, Section 2.2) shows that when participants are offered an explicit option to randomize between the options, they choose it in $53 \%$ of cases. The frequency of choosing such coin flipping is even higher than in a control treatment where the same options are made available through a repeated-choice format like the one in Experiment I. Finally, Experiment III (Section 2.3p tests whether reversals can also appear in situations where people have to rank more than just two options. The data analysis of Experiment III also rejects that subjects who reverse the order of the prizes are simply indifferent between them.

Two experimental literatures have previously generated evidence on a preference for randomization. First, procedural concerns have been studied extensively in the context of fairness. People do care for procedures, not just for outcomes. For example, they have preferences over different allocation mechanisms even if they are not participating in the mechanism (Kahneman et al. 1986). Famously, the procedure becomes more important than the outcome in the example of "Machina's mom" (Machina 1989). She decides to flip a coin in order to decide which of her two children gets an indivisible goody although she has a preference for one of the children getting it rather than the other child. Flipping the coin is more preferable to her (or rescues her from bearing the consequences of showing her preference order). Experimental results confirm that people may prefer ex ante fair lotteries if an ex post fair outcome is unavailable (Bolton et al. 2005). In a similar vein, Krawczyk and Le Lec (2010) investigate dictator games with a limited strategy set and show that subjects allocate a positive probability to the outcome that the other player gets the whole payoff: $]^{5}$ Finally, Bohra (2010) develops a model where a decision maker's utility is a weighted sum of her expected utility from the lottery outcome as well as a procedural component.

The second set of related experimental studies concerns the betweenness axiom and the

\footnotetext{
${ }^{5}$ Sen (1997) proposes to accommodate concerns for the procedure in menu-dependent models where preferences over outcomes can depend on the menu or set of outcomes available.
} 
possibility of false diversification effects. The betweenness axiom stipulates that if a decision maker prefers $\mathrm{X}$ to $\mathrm{Y}$ she must also prefer $\mathrm{X}$ to a mixture between $\mathrm{X}$ and $\mathrm{Y}$ and the mixture to Y. Camerer and Ho (1994) report on experiments where the betweenness axiom is often violated. The main difference of these experiments to our setup is that our subjects choose between two safe options and a lottery between the two safe options while in the betweenness experiments subjects choose between three lotteries with two to three possible outcomes each. Thus, our setup is simpler with respect to the lotteries involved, focussing on the preference for random versus safe outcomes. In the experiment of Chen and Corter (2006), the participants are asked to state the same decision between a safe and a risky outcome for a number of repeated decisions. In the experiment's single-play condition, participants choose between a sure gain of $\$ 5$ and a $50 \%$ chance to win $\$ 15$. In the multiple-play condition they choose ten prospects, where each of them is either a sure gain of $\$ 5$ or a play of the gamble that consists of a $50 \%$ chance to win $\$ 15$. It is found that people tend to prefer a mix of the risky and the risk-free option in the multiple-trial choices. This contradicts expected utility but not betweenness. It can be viewed as stemming from a false belief in the diversification value that arises from mixtures, like in the field experiment of Read and Loewenstein (1995). In contrast to these studies, our participants can get at most one prize, erasing the possibility of a diversification value. Thus, a false belief in diversification would have to be quite false and cannot serve as a likely explanation of behavior. Rubinstein (2002), however, has documented a false diversification of experimental choices in a context where only one prize can be earned. He confronts his participants with several decisions between lotteries with known probabilities and finds irrational mixtures of choice: his participants, just like ours, violate stochastic dominance. But while Rubinstein (2002) ascribes the effect to a cognitive failure of grasping the multi-stage randomness in his experiment (wrongly applying the intuition of diversification), the experiments in this paper are much simpler than his tasks and we can assume them to be fully understood by the participants. Therefore, our preferred 
interpretation of the data is one where the participants know what they are doing and want to do it.

Another theory where procedures can affect utility, not just outcomes, is the theory of regret (Loomes and Sudgen 1982). According to this theory, decision-makers anticipate that they will experience disutility from having made an ex-post suboptimal decision. Their choice aims (among other things) at minimizing regret. To apply regret theory, one needs to assume that a certain degree of uncertainty prevails regarding the utility stemming from the decision. This is highly plausible in the context of university admission but somewhat less plausible in the case for vouchers that are valid in well-known stores. We also note that for our choice contexts, standard regret theory alone is not sufficient to explain a preference for randomization because not even with randomization can participants avoid decisions that turn out to be wrong afterwards. Depending on the outcome of the chance move, they can still receive a suboptimal outcome. (The chance of experiencing regret is even larger than if they choose a nonrandom strategy.) But it is conceivable that subjects experience less regret if they can blame the random draw, in cases where the random draw instead of their own choice generates the wrong action. If augmented with this feature, regret aversion is a potential explanation for preference reversals. Just like the evidence on preference for procedural fairness discussed above, the hypothesis that decision-makers prefer to involve a random draw points at a fundamentally procedural choice motive: some decision-makers prefer to avoid the decision even if this comes at the cost of a (probabilistic) material consequence. In the context of university choice, the hypothesis translates into the possibility that applicants may prefer not to make a firm choice themselves but prefer for their applications to account for the different pros and cons of the different universities.

The remainder of the paper proceeds as follows. In Section 2, the series of classroom experiments is described together with their results. Section 3 introduces the empirical context of university choice in Germany. To identify the subset of data that is suitable for our purposes, 
the section then discusses and applies the relevant literature on matching procedures. Finally, it shows the calculations that yield our main empirical result. Section 4 concludes.

\section{Experiments}

In this section, we introduce the experimental design and present the results. All experiments were run outside of the laboratory as the one-shot individual decision task can be investigated more efficiently in other settings. We employed classroom experiments as well as a newspaper experiment in combination with an online survey. While the subjects in the classroom experiment are students, a more diverse subject pool is reached with the newspaper experiment.

The recruitment of participants differed between the three experiments. The classroom experiments (Experiment I, Experiment II and parts of Experiment III) were conducted in introductory lectures in microeconomics and macroeconomics for undergraduate students at the Technical University Berlin and at the Free University Berlin. The newspaper experiment (part of Experiment III) was advertised in the "WZB-Mitteilungen", a quarterly journal of the Social Science Research Center Berlin (WZB), which is read by researchers, policy makers and the interested public. No participant took part in more than one experiment or more than one treatment 6

\subsection{Experiment I: deliberate choice reversals}

\subsubsection{Design}

In the first experiment, students were given the choice between two prizes. One prize ("alternative A") was a 19 Euros Starbucks gift voucher. The other prize ("alternative B") was a 14

\footnotetext{
${ }^{6}$ All instructions are available from the authors upon request.
} 
Euros Starbucks voucher together with a 10 Euros Amazon voucher. Thus, the participants had to consider multiple dimensions to make their choice (the amount of money and the types of goods that can be obtained with a voucher). Participants had to decide twice which prize they preferred, but each of the two choices was realized only with a certain probability. In particular, they were told that they would receive the prize chosen in the first task with a probability of .6 and the prize chosen in the second task with a probability of .4 .

In addition, a control treatment was conducted in which (a separate set of) participants were told that they could choose one of the two alternatives and that they would receive this prize with probability .4. This treatment serves to control for the possibility that due to the lower probability of receiving a prize in the second task in the main treatment, the less preferred choice is chosen more often because subjects expect to get it with a lower probability. Such a prediction would result from various models of stochastic choice, e.g. the logit model. Both treatments were run in parallel in the same classrooms, by giving out different sets of instructions to different students.

We payed out a prize to every tenth participating student. Thus, at the end of the experiment, we randomly determined the participants who received a prize and played out the random draws for the two treatments.

\subsubsection{Results}

Of the 69 participants in the treatment group, $23 \%$ chose $\mathrm{A}$ and $77 \%$ chose $\mathrm{B}$ in the first task. In the second task, participants chose $\mathrm{A}$ in $36 \%$ and $\mathrm{B}$ in $64 \%$ of the cases. Thus, the majority of participants prefers B over A, but it is also evident that some subjects took different decisions in the two tasks. When investigating the choices in the two tasks in the treatment group together, we observe that $28 \%$ of participants chose two different alternatives in the two tasks. Thus, they either chose A in the first and B in the second task or the reverse. 
RESULT 1: Almost one third of the participants (28\%) submit two different decisions in the two decision tasks.

One possible explanation for this choice pattern is that subjects choose their less preferred option more often when the probability of receiving it is relatively low. For example, a model of random utility, leading to payoff sensitive choice probabilities like in logit choice, could accommodate the observed patterns in the treatment group. If this explanation is correct, then the choices in the control treatment should be the same as the choices in the second task of the main treatment, as the prizes are chosen with the same probability in both treatments. However, this null hypothesis is rejected. While in the second task of the main treatment, $36 \%$ opt for alternative A, only $19 \%$ choose A in the control treatment $(N=68)$. The difference is statistically significant (Fisher exact test, $p=0.02$ ).

RESULT 2: The observation that individuals submit different decisions cannot be explained by random utility models. The presence of the first choice problem significantly affects the choice probability in the second choice problem.

\subsection{Experiment II: explicit preference for randomness}

\subsubsection{Design}

In this experiment, participants again had the choice between two prizes. Alternative A was a 19 Euro Starbucks voucher and alternative B a 19 Euro Amazon voucher. Two treatments were conducted, varying the choice format. In the binary-choice treatment, the participants were asked to choose between the two alternatives and, just as in Experiment I, they had to make this choice twice. The first choice became relevant with a probability of .6 and the second choice with a probability of .4. In the second treatment, the four-way-choice treatment, participants faced the choice between four options: 
Option 1: You receive alternative $\mathrm{A}$ with a probability of $60 \%$ and alternative $\mathrm{B}$ with a probability of $40 \%$.

Option 2: You receive alternative A with a probability of $100 \%$.

Option 3: You receive alternative B with a probability of $60 \%$ and alternative A with a probability of $40 \%$.

Option 4: You receive alternative B with a probability of $100 \%$.

Here and in all other experiments in this paper, the probabilities were presented as percentage points. In the experimental instructions, we provided a description of the probabilities in terms of the frequency that a certain event occurs when 100 draws from an urn are made. Just as in Experiment I, subjects were told that at the end of the experiment, every tenth participant would be paid and would receive the option that he or she had chosen. The probabilities were played out by the computer to determine the relevant choices and the outcomes of the lotteries.

\subsubsection{Results}

166 students participated in the classroom experiment. Of these 166 students, 74 participated in the binary-choice treatment and 92 participated in the four-way-choice treatment. In the four-way choice treatment, $53 \%$ chose the explicit option to randomize between the two different alternative prizes, i.e., option 1 or option 3 . In the binary-choice format, only $28 \%$ of students randomized by choosing both $\mathrm{A}$ and $\mathrm{B}$ exactly once in the two questions. The difference between treatments is statistically significant $\left(p<0.01\right.$, two-sided Pearson's $\chi^{2}$ test).

RESULT 3: When subjects are given the opportunity to choose a lottery between two alternatives, they choose a random outcome more often than in an equivalent repeated-choice 
format where they can only randomize indirectly by choosing different alternatives in the different tasks.

\subsection{Experiment III: reversals between longer preference lists}

In Experiments I and II, there were only two alternatives among which participants could choose, differently from many real-life settings such as university choice where decision-makers have to rank more than two alternatives. This raises the question whether reversals in the rank order can also be found in choice between longer lists. A third series of experiments was conducted to address this issue. Experiment III also uses a different trick (different from Experiment I) to rule out that preference reversals are simply due to indifference.

\subsubsection{Design}

In this third set of experiments, a classroom and a newspaper experiment, there were four available prizes A to D which consisted of combinations of gift vouchers and/or cash. Table 1 shows the prizes for both experiments.

Table 1: Prizes to be ranked in the experiments

\begin{tabular}{lll}
\hline \hline & Classroom & Newspaper \\
\hline A & 19 Euros Starbucks gift voucher & 54 Euros Amazon gift voucher \\
B & 19 Euros Amazon gift voucher & 42 Euros in cash \\
C & 14 Euros Starbucks and 10 Euros Amazon gift & 34 Euros Amazon gift voucher and 12 Euros in \\
& vouchers & cash \\
D $\quad$ 8 Euros Starbucks and 16 Euros Amazon gift & $\begin{array}{l}14 \text { Euros Amazon gift voucher and 30 Euros in } \\
\text { vouchers }\end{array}$ \\
\hline \hline
\end{tabular}

Prizes in the newspaper experiment were slightly higher in order to motivate readers to participate by going to a webpage and submitting their preferences there. Since readers of the "WZB-Mitteilungen" may be less likely to go to Starbucks than students, we replaced the Starbucks vouchers by cash payments.

Participants were asked to rank the prizes on two lists, list (i) and list (ii), where each 
list is chosen to be payoff-relevant with a probability of .5. Each list, if chosen, delivers the differently ranked prizes with given probabilities. On both lists, the prize ranked first is received with the highest probability, the prize ranked second with the second highest probability etc. As shown in Table 2 , the distribution of probabilities among the different ranks slightly differs across the two lists.

Table 2: Probabilistic consequences of the two preference lists

\begin{tabular}{lll}
\hline \hline Probability of getting prize ranked & List (i) & List (ii) \\
\hline 1st & .5 & .4 \\
2nd & .3 & .3 \\
3rd & .2 & .2 \\
4th & 0 & .1 \\
\hline \hline
\end{tabular}

An expected-utility maximizer with strict preferences over the available prizes A to D would list her most preferred prize first on both lists, the second preferred prize second etc. Any other combination of lists would violate stochastic dominance.

However, if a decision maker is indifferent between, say, prize $\mathrm{B}$ and prize $\mathrm{C}$, it is consistent for her to list prize B first on list (i) and prize C first on list (ii). To address the possibility that reversals are due to indifference, we added a (surprise) second part of the experiment, appearing immediately after the participants submitted their preferred lists. In the second part, participants were asked to simply pick their most preferred prize (A, B, C, or D). (Only one of the two parts was payoff-relevant, as detailed below.) We then investigate whether the subset of decision makers who top-ranked two different prizes on list (i) and list (ii) chose the two prizes with equal probability in the second part of the experiment. This would be natural to expect under the hypothesis of indifference between alternatives. On the other hand, if subjects are not indifferent but have a strict preference of one prize over the other, then it can be expected that they list the preferred prize on list (i) at first rank where the probability to receive it is higher than on list (ii) 7 That is, in case of a deliberate reversal at the first

\footnotetext{
${ }^{7}$ List (i) also appeared before list (ii) on the decision sheets/screens, making it plausible that the "true" top-ranked alternative would be listed on list (i).
} 
preference rank, part II choices will tend to confirm list (i), and not list (ii).

In both the classroom and the newspaper experiments the prize in part I of the experiment was determined according to the probabilities listed in Table 2. Concerning part II, participants received their chosen prize. However, not every participant received a prize. In the classroom experiment, 10 percent of participants received a prize in part I and a different set of 10 percent of participants received a prize in part II. In the newspaper experiment, there was an ex-ante fixed number of ten winners for part I and ten different winners for part II, to be drawn randomly. The payoff rules were explained to all participants in details, in order to minimize the chance that preference reversals would result from non-preference motives like a false belief in diversification.

\subsubsection{Results}

In the classroom experiment, a total of 314 students participated. 126 of them (40\%) stated different preferences on lists (i) and list (ii). The differences between the lists follow discernible patterns. Almost all reversals $(92 \%)$ involve adjacent ranks. Thus, in most cases subjects switch the ranking of two prizes that are next to each other on the list. Furthermore, 99 out of the 126 reversals (79\%) occur at the first rank of the lists. Of these 99 occurrences, 56 participants confirmed list (i) in part II, 30 confirmed list (ii), and 13 participants confirmed neither list (i) nor list (ii) in part II. The difference in confirmations of list (i) and list (ii) is significant $(p<0.01$, one-sided Binomial test with $N=86) 8$ which is consistent with our hypothesis that participants are not exactly indifferent between choice options, but deliberately bias their entries on list (ii).

\footnotetext{
${ }^{8}$ Note that alternatives here are trichotomic: confirm list (i), confirm list (ii), confirm none of the two lists. However, a Binomial test (on confirming list (i) versus confirming list (ii)) can still be used if the third group (that does not confirm any list) is made up fifty-fifty by the two former groups in expectation. In that case, the number of observations relevant for the test equals the total number of reversals less the number of participants not confirming any list. Since there is no reason to assume that the third group is selective, this is how we proceed. We therefore have $N=99-13=86$.
} 
In the newspaper experiment, 194 participants submitted a complete set of online responses. As the "WZB-Mitteilungen" is distributed to social scientists, policy makers and other professionals, the subject pool differs markedly from the students in the classroom experiments. About half of the participants were directly affiliated with the WZB and thus either social scientists or familiar with research in social sciences.

In the newspaper experiment, 29 participants (15\%) did not submit identical lists. This percentage is lower than in the classroom experiments, but the structure of reversals is again similar. We observe that in 28 out of 29 reversals (97\%) only adjacent ranks are involved. Of the 29 reversals, 15 occur at the first rank (52\%). Regarding the hypothesis that choices in part II of the experiment should confirm the first choice on list (i), we find supportive evidence. In part II, ten participants confirmed list (i) and only three confirmed list (ii). This difference is again statistically significant $(p<0.05$, one-sided Binomial test with $N=13) ! 9$ i.e., participants do not randomize because of indifference between alternatives but instead deliberately reverse their preferences. We summarize our findings from above in Result 4:

RESULT 4: $40 \%$ of participants in the classroom experiment and 15\% of participants in the newspaper experiment submit different rank-order lists. The majority of participants in both experiments with reversals at rank 1 confirmed their first choice of list (i) as their most preferred choice in part II of the experiment, rejecting the hypothesis that they are indifferent between two choices and randomize between them with equal probability.

\section{Empirical evidence: reversals in university applications}

The experiments we have discussed thus far are extremely simple, which has the advantage of allowing us to study preference reversals in a setting uncluttered with confounding factors.

\footnotetext{
${ }^{9}$ As above the number of observations for the test equals the total number of reversals occurring at rank one less the number of participants not confirming any list, i.e., 15-2=13.
} 
On the other hand, the experiments only involve relatively small stakes for participants, so that subjects might not give a lot of thought to the decisions they are taking. In this section we report real life evidence on an important decision in people' lives: university applications. We show that the results we obtained in stylized experiments carry over to this context, in the sense that a non-negligible frequency of violations of stochastic dominance occurs. We also demonstrate that the consequences of this decisions are substantial.

\subsection{Admission to university in Germany}

Under the German system, university admissions for medical subjects are centrally administered by a central clearinghouse. The central clearinghouse assigns applicants according to the following three procedures that are implemented in a sequential order:

(1) Procedure A admits students who are top of the class to around $20 \%$ of seats.

(2) Procedure $\mathrm{W}$ admits students with long waiting times to around $20 \%$ of seats.

(3) Procedure U represents admission by universities according to their own criteria to around $60 \%$ of seats.

For each of the three procedures, applicants are asked to submit a preference ranking of universities, which might either be identical or different across procedures. All rank-order lists are made at the same moment in time. The central clearing house employs the three procedures in a strictly sequential order: all applicants who are matched in procedure A are firmly assigned a seat at their matched university and do not take part in subsequent procedures. All remaining applicants enter procedure W. Likewise, after procedure W, all applicants who are still unmatched enter procedure $\mathrm{U}$. The fact that applicants can submit three (potentially different) rank-order lists of universities, each of which may be relevant, is a unique property of the German mechanism, suitable for our analysis. In the following, we will use the lists to infer true preferences and to compare the lists that are effectively submitted, in order to 
measure the frequency of preference reversals, i.e., the submission of different preference lists by the same applicants. But not all instances of self-contradicting applications is necessarily a violation of stochastic dominance. Studying the incentives of applicants in detail (and applying the literature on matching algorithms) we will identify the subgroups of applicants who are most relevant in our context: applicants for whom a preference reversal not only may have a material consequence but who also have no "rational" incentives to reverse their preferences. For these individuals, a reversal of preferences amounts to a stochastic-dominance violation. Under the assumption that they have strict preferences over their possible places of study (which we regard as reasonable, given the decision's importance), a reversal made by these individuals increases the probability of ending up at a suboptimal university.

Because applicants who are successful in procedure $\mathrm{W}$ are a disjoint group with poor grades and low chances of being admitted through the other two procedures, we restrict our attention to procedures $\mathrm{A}$ and $\mathrm{U}$, where admission largely follows grade point average (GPA) in final secondary-school examinations. For both procedures A and U, applicants are allowed to rank no more than six universities ${ }^{10}$ In the following paragraphs, we will briefly introduce the two procedures and the relevant matching literature, before we move on to the description of the data set and to the results.

\subsubsection{Procedure U}

For expositional reasons, we will start with introducing procedure $\mathrm{U}$ even though it is administered at the end of the admission process. In procedure $\mathrm{U}$, universities are given the opportunity to select the majority of their students themselves, that is, procedure $U$ corresponds to a two-sided market where both students and universities have preferences over their match. For admission each university ranks the applicants who have listed it, using

\footnotetext{
${ }^{10}$ Until the winter term 2004/05 applicants could rank seven universities; to be consistent over time we only consider the first six ranks. For a more detailed description of the admission process see Braun et al. (2010) and Braun and Dwenger (2009).
} 
the final grade from school as the predominant, but not necessarily the only criterion. Given the preference lists of universities and students, the central clearinghouse applies the collegeproposing Gale-Shapley algorithm on the applicants. The algorithm was first described by Gale and Shapley (1962) although similar ideas had been in use since the 1950s in the U.S. clearinghouse for the first jobs of doctors (see Roth 2008). A more detailed description of the college-proposing Gale-Shapley mechanism can be found in the appendix.

We assume truth-telling under procedure U, i.e., we use the rank-order lists submitted for procedure $\mathrm{U}$ to infer the (possibly incomplete) true preferences of the applicants. This assumption is justified not least because the information brochure of the clearing house advises applicants explicitly to reveal their preferences under procedure U. Importantly, this advice is normatively justified as there are theoretical reasons why the submitted lists under procedure $\mathrm{U}$ should reflect the true preferences, at least in an incomplete sense. First, in the collegeproposing Gale-Shapley mechanism all successful manipulations can also be accomplished by truncations (see Roth and Peranson (1999), p.762, referring to results by Roth and Vande Vate (1991)). Thus, even if applicants strategically truncate their lists submitted in procedure $\mathrm{U} 11$ the correct rank order of the remaining choices is preserved. And since truncations are the manipulations that require the least information about others' preferences, they are more likely to occur than other manipulations (see Roth and Rothblum 1999).

Second, if preferences of universities are perfectly correlated (which they are if universities rank applicants only by their final grades from school), then there is only one stable matching 12 In this case, the stable matching is achieved by both the college- and the studentproposing Gale-Shapley mechanism. Since the latter is strategy-proof (see Roth 1982), it then

\footnotetext{
${ }^{11}$ In the information brochure, the central clearinghouse does not mention the possibility of such truncations or other forms of strategically misrepresenting one's true preferences in procedure U (except for a preselection stage which does not affect our analysis).

${ }^{12}$ In a stable matching everybody prefers their match over no match at all and there is no applicant and university who are not matched but who would both prefer to be. To see why there is only one stable matching if university preferences are perfectly correlated, note that in any stable matching the applicant ranked highest by the universities gets his preferred matching. Thus, there is only one stable matching for the applicant with the best final grade. This argument can be repeated for all other applicants.
} 
follows that truth-telling is also a dominant strategy in the college-proposing Gale-Shapley mechanism. Thus, we can conclude that the incentives to misrepresent one's preferences in procedure $\mathrm{U}$ are null for perfectly correlated preferences of universities and they are very small if the preferences of universities are strongly correlated. This is the case in the German university admission system where all universities have to use the GPA as the main criterion due to legal constraints and where some universities even base their ranking of applicants solely on the final grade.

To sum up, we can use the preference ranking over universities submitted for procedure $U$ to infer an applicant's true preferences. In our data analysis, we will compare an applicant's true preference list to the rank-order list that she submitted for procedure A.

\subsubsection{Procedure A}

Procedure A is employed to reward excellent GPAs in secondary schools. Applicants with the best average grades are selected and then assigned according to the preference list they submitted for procedure A. To this end, the "Boston mechanism" is applied, which assigns as many applicants as possible to their first choice ( $k^{\text {th }}$ choice) and considers second choices $\left((k+1)^{t h}\right.$ choices) only if there are still seats left at the end of the first $\left(k^{t h}\right)$ round (cf. the appendix for details on the Boston mechanism).

The Boston mechanism implies that an applicant ranking a university in $k^{\text {th }}$ position is admitted before applicants ranking a university in $(k+1)^{\text {th }}$ position are considered - independently of her high-school GPA. Hence, it may be advantageous for some applicants to manipulate their true preference ordering by skipping a university if they do not have any chance of getting a seat. If applicants understand the strategic incentives of the mechanism, this would lead us to overstate the share of applicants deliberately changing their preference ordering. 
In addition to the strategic incentives set by the Boston mechanism itself, the sequential nature of the admission process creates incentives to misrepresent one's preferences in procedure A. Procedure A is the first to be administered. As assignments in each of the procedures are fixed, only applicants who have not been admitted through procedure A (or through procedure $\mathrm{W}$ ) participate in procedure $\mathrm{U}$. Both procedures A and U largely follow average grades, that is, applicants with very good GPA have a chance to be admitted in both procedures. These individuals should avoid being matched to a less preferred university in procedure A and wait for procedure $\mathrm{U}$ where they have a very good chance to be admitted to one of their top choices. Applicants therefore might submit rank-order lists of universities in procedure $\mathrm{A}$ that are shorter than their true preference order ${ }^{13}$ Due to the strategic behavior of applicants, we potentially overstate the share of applicants not ranking a university in procedure A but in procedure $\mathrm{U}$.

In our analysis below, we check whether preference reversals can be driven by these strategic motives of the applicants. By eliminating these observations, we identify pure reversals in the sense that applicants appear to express a preference for randomization.

\subsection{Description of the data}

We use the (anonymized) information collected by the central clearinghouse covering seven waves of applications between winter term 2005/06 and winter term 2008/09. During our observation period the following six subjects were centrally administered and are part of our data set: biology, medicine, pharmacy, psychology, animal health, and dentistry. The data set contains all applications for these subjects and records all information provided by the applicants including data on individual characteristics such as final GPA, age, sex, and place of living. Most important for our purpose, the database provides information on the admission

\footnotetext{
${ }^{13}$ Braun et al. (2010) demonstrate that many applicants react insufficiently to this incentive and submit too long lists. The objective of procedure A-to give an additional advantage to the applicants with highest GPA-therefore fails to be met.
} 
procedures that a prospective student has participated in as well as his or her rank-order lists on universities for the different procedures.

To avoid multiple counting, we only consider each individual's first application in the data set. After excluding individuals not applying for procedure $\mathrm{U}$ and discarding individuals whose preference list for procedure $\mathrm{U}$ might be incomplete due to preselection ${ }^{14}$ we are left with a total number of 224,016 first-time applications.

\subsection{Results: preference reversals in university applications}

We first consider the full data set as described. In line with the evidence found in our experiments, we observe many individuals reversing the order of their preference rankings over universities. We then subject our findings to several robustness checks. To rule out that strategic manipulations by the applicants drive our results, we focus on a restricted data set, which only contains individuals who do not face any incentive to strategize (restriction I, see Section 3.1.2). We also show the robustness of our results with regard to the exclusion of applicants who might anticipate that they have no reasonable chance of being admitted in one of the procedures analyzed and who, for that reason, might not well-consider their preference lists (restriction II).

\subsubsection{Full data set}

We examine the full set of 224,016 first-time applications to study whether preference lists coincide in procedures A and U. Table 3 shows where the entries on the lists submitted for

\footnotetext{
${ }^{14}$ All procedures are two-stage procedures. At the first stage, applicants are preselected ("preselection"). At the second stage, the selected applicants compete for admission to one of their preferred universities ("admission"). Universities can delegate preselection to the clearinghouse, which then shortlists applicants according to the preference rank the applicant has given to the university and according to GPA. The preselection criteria applied by the universities differ, requiring for example that the university be listed as a first or first to third preference. Some universities use a combination of average final grade and preference rank. Importantly, after preselection the applicants are allowed to reshuffle the ordering of their rank-list. We only consider the final rank-order list for procedure $\mathrm{U}$ as this list should be free from strategic manipulations.
} 
procedure $\mathrm{U}$ appear as entries for procedure A. Row $i=1 \ldots 6$ of the table contains, for the universities on rank $i$ for procedure $\mathrm{U}$, the percentage of cases in which the same applicant listed the university on rank $j$ of the list for procedure A.

Table 3: Conditional proportions, showing where universities ranked in procedure U appear in rank-order lists for procedure A (all entries in \%)

\begin{tabular}{c|rrrrrrr|r}
\hline \hline $\mathrm{U} / \mathrm{A}$ & 1 & 2 & 3 & 4 & 5 & 6 & not ranked & total \\
\hline 1 & 75.427 & 4.052 & 1.911 & 1.135 & 0.780 & 0.543 & 16.153 & 24.574 \\
2 & 5.337 & 63.407 & 4.825 & 2.366 & 1.641 & 1.098 & 21.326 & 37.475 \\
3 & 2.710 & 5.227 & 58.806 & 4.758 & 2.839 & 1.778 & 23.883 & 41.195 \\
4 & 2.347 & 3.453 & 5.774 & 55.884 & 4.940 & 2.637 & 24.964 & 44.115 \\
5 & 1.743 & 2.358 & 3.416 & 5.873 & 55.228 & 4.588 & 26.794 & 44.772 \\
6 & 1.658 & 1.886 & 2.360 & 3.523 & 5.452 & 54.918 & 30.203 & 45.082 \\
\hline \hline
\end{tabular}

Notes: Full data set as described in Section 3.2 ( $N=224,016$ observations).

Source: Own calculations based on ZVS data on applicants, waves 2005/06 to 2008/09.

The diagonal elements in the table indicate unchanged preferences. For example, in $75 \%$ of applications the top-ranked university from procedure $\mathrm{U}$ is also ranked first in procedure A. Similarly, $63 \%$ of universities ranked on rank two in procedure $U$ are equally ranked in procedure A, etc. The off-diagonal elements report the discrepancies between the lists that applicants submit in the two procedures A and U. Discrepancies at a certain preference rank can result either from listing different universities or from not listing any university on a given rank of one list, but listing a university on the same rank of the other list. The results in Table 3 show that a considerable number of subjects submit different lists. Every fourth applicant reverses the university that she top-ranked in procedure $U$ with a lower rank in procedure A or does not rank it at all. The relatively large table entries that appear adjacent to the diagonal indicate another pattern: many preference reversals are formed by moving a university to an adjacent preference rank.

Notice that skipping the top-ranked university in procedure A moves up all universities named on rank 2 and below. In Table 3 skipping the top-ranked university is therefore recorded as a number of preference reversals further down the list. To avoid such double counting, Table 4 repeats the counting exercise of Table 3 but only includes the first preference 
reversal within an application. Entries in rows $2 \ldots 6$ of Table 4 (marked with a star) reflect additional cases of preference reversals, relative to previous ranks. Not ranking the top university from procedure U in procedure A thus only appears in the first row as "not ranked" while the effects of this reversal further down the list are not counted. Table 4 therefore counts each application with a preference reversal exactly once. It shows that preference reversals are widespread among individuals applying for a seat at university: a total of $64 \%$ of all applicants reverse at least one preference rank between procedures $\mathrm{U}$ and $\mathrm{A}$ or do not list a university in procedure A that is listed in procedure U. Disregarding reversals from not ranking universities in procedure A but in procedure $\mathrm{U}(36 \%)$ shows that approximately $29 \%$ of applicants change the ordering of their preference lists.

Table 4: Preference reversals (all entries in \%)

\begin{tabular}{c|rrrrrrr}
\hline \hline $\mathrm{U} / \mathrm{A}$ & 2 & 3 & 4 & 5 & 6 & not ranked & total \\
\hline 1 & 4.052 & 1.911 & 1.135 & 0.780 & 0.543 & 16.153 & 24.574 \\
$2^{*}$ & - & 4.576 & 1.924 & 1.158 & 0.779 & 7.674 & 16.111 \\
$3^{*}$ & - & - & 3.554 & 1.582 & 0.855 & 3.885 & 9.876 \\
$4^{*}$ & - & - & - & 2.868 & 1.115 & 3.022 & 7.005 \\
$5^{*}$ & - & - & - & - & 1.800 & 2.213 & 4.013 \\
$6^{*}$ & - & - & - & - & - & 2.861 & 2.861 \\
total & 4.052 & 6.487 & 6.613 & 6.388 & 5.092 & 35.808 & 64.440 \\
\hline \hline
\end{tabular}

Notes: See Table 3

RESULT 5: 29\% of university applicants change the ordering in which universities appear on their rank-order lists across procedures. Another $36 \%$ of applicants do not list a university in procedure $A$ but list it in procedure $U$.

\subsubsection{Restriction I: ruling out strategic manipulations}

As noted in Section 3.1.2, applicants may have incentives to skip a university in the list for procedure A if they expect not to have any chance of being admitted. They may also truncate their rank-order list for procedure A in order to avoid being matched to a lower preference in procedure A and instead wait for procedure U. In order to act on these incentives, applicants need to anticipate their chances to be matched to a given university if it is listed on a given rank 
for procedure A. The central clearinghouse publishes detailed information on the application characteristics of admitted candidates for every university-field combination in each year. It also makes the applicants aware that they should use the historical admissions statistics when devising the list for procedure A, and points out the potential advantage of manipulating the list. Under the auxiliary assumption that admission chances are stable from year to year, the applicants have the information that is available for strategic manipulations and they will plausibly use them. To exclude all observations where strategic considerations of the applicants can be at work, summarized as "restriction I", we first focus on applicants who, on the considered rank, have a chance to be admitted in procedure A to the university listed in procedure U. Second, we restrict attention to ranks where a university is listed in both procedures. 91,485 first-time applications in our data set fulfill this restriction.

Table 5: Conditional proportions after restriction I, ruling out strategic incentives (all entries in $\%$ )

\begin{tabular}{c|rrrrrrr|r}
\hline \hline $\mathrm{U} / \mathrm{A}$ & 1 & 2 & 3 & 4 & 5 & 6 & not ranked & total \\
\hline 1 & 87.094 & 4.993 & 2.429 & 1.435 & 0.996 & 0.696 & 2.357 & 12.906 \\
2 & 6.512 & 71.389 & 6.457 & 3.192 & 2.357 & 1.593 & 8.500 & 28.611 \\
3 & 2.959 & 6.032 & 66.006 & 6.199 & 4.028 & 2.489 & 12.286 & 33.993 \\
4 & 3.099 & 3.916 & 6.725 & 62.100 & 6.473 & 3.674 & 14.012 & 37.899 \\
5 & 1.953 & 2.639 & 3.886 & 6.507 & 63.007 & 5.987 & 16.021 & 36.993 \\
6 & 1.715 & 2.186 & 2.946 & 4.385 & 6.236 & 61.883 & 20.649 & 38.117 \\
\hline \hline
\end{tabular}

Notes: We only consider applications where both preference rankings contain a nonempty cell in the considered rank, and where the applicant's entry in procedure U would have been successful in the previous year, conditional on previous ranks being unsuccessful. $N=91,485$ observations.

Source: Own calculations based on ZVS, data on applicants, waves 2005/06 to 2008/09.

In Table 5 the fraction of applications stating a university on the same rank in procedure A as in procedure U increases: $87 \%$ compared to $75 \%$ (Table 3) of applicants top-rank the same university in procedures $\mathrm{U}$ and $\mathrm{A}$. In particular, the proportion of universities that are top-ranked in procedure $\mathrm{U}$ but not ranked in procedure A drops significantly from $16 \%$ (Table 3) to $2 \%$. This is exactly what we would expect if applicants strategically skip their first preference in procedure A because they do not expect to have a chance of getting a seat. On lower ranks, the difference in the proportions of applicants not ranking a university in procedure A named in procedure $\mathrm{U}$ is still significant but smaller than in the top ranks. 
This, too, is to be expected from rational applications, as the Boston Mechanism's strategic incentives to skip a university are greater for top ranks. The evidence is generally consistent with the hypothesis that part of the preference reversals found in Tables 3 and 4 are due to strategic considerations of the applicants. Yet, after applying restriction I the incentives are muted but we still see a large proportion of individuals reversing their rank-order lists. Table 6 summarizes the results of Table 5 without double counting further down the list (analogous to the transition from Table 3 to Table 4); $28 \%$ of applicants in procedure A either do not rank a university listed in procedure $\mathrm{U}(7 \%)$ or swap preferences $(21 \%)$.

Table 6: Preference reversals after restriction I, ruling out strategic incentives (all entries in $\%)$

\begin{tabular}{c|rrrrrrr}
\hline \hline $\mathrm{U} / \mathrm{A}$ & 2 & 3 & 4 & 5 & 6 & not ranked & total \\
\hline 1 & 4.993 & 2.429 & 1.435 & 0.996 & 0.696 & 2.357 & 12.906 \\
$2^{*}$ & - & 3.219 & 1.382 & 0.847 & 0.553 & 1.721 & 7.721 \\
$3^{*}$ & - & - & 1.635 & 0.732 & 0.407 & 1.024 & 3.798 \\
$4^{*}$ & - & - & - & 0.956 & 0.402 & 0.603 & 1.962 \\
$5^{*}$ & - & - & - & - & 0.447 & 0.394 & 0.841 \\
$6^{*}$ & - & - & - & - & - & 0.468 & 0.468 \\
total & 4.993 & 5.648 & 4.452 & 3.531 & 2.505 & 6.567 & 27.696 \\
\hline \hline
\end{tabular}

Notes: See Table 5

RESULT 6: After removing applicants who have an incentive to submit different rank order lists, 28\% of applicants display preference reversals between lists. 13\% of applicants submit pairs of preference orders where a different university appears on rank 1.

\subsubsection{Restriction II: ruling out irrelevance}

When submitting their preference lists, applicants do not know for certain whether they will be selected for any of the procedures. But using the historical admissions data, they can assess the chances with which each of the procedures applies to them. A natural possibility is that applicants sometimes do not follow their true preferences when filling out lists in procedures that are likely not relevant for them. This may result in preference reversals that are solely due to the fact that one list is likely irrelevant: e.g., an applicant who can be sure to matched 
under procedure A (because her GPA is clearly good enough for her top choice on list A) might as well report a reversed list for procedure U. To rule out irrelevance ("restriction II"), we drop individuals from the data set whose GPA is at least 0.4 grade points better than the threshold needed for admission on the considered rank of list A. Further, we focus on applicants who belong to the (top 20\%) group that is selected for procedure A to make sure that preference list $\mathrm{A}$ is well-considered.

Table 7: Conditional proportions after restriction I and II, ruling out strategic incentives and irrelevance (all entries in \%)

\begin{tabular}{c|rrrrrrr|r}
\hline \hline $\mathrm{U} / \mathrm{A}$ & 1 & 2 & 3 & 4 & 5 & 6 & not ranked & total \\
\hline 1 & 93.665 & 3.385 & 0.893 & 0.376 & 0.270 & 0.153 & 1.258 & 6.335 \\
2 & 1.904 & 74.760 & 3.828 & 1.294 & 0.647 & 0.333 & 17.234 & 25.240 \\
3 & 0.879 & 3.345 & 66.138 & 3.418 & 2.051 & 0.903 & 23.266 & 33.862 \\
4 & 0.643 & 1.930 & 3.799 & 61.520 & 3.615 & 1.501 & 26.992 & 38.480 \\
5 & 0.228 & 0.456 & 1.674 & 3.956 & 61.126 & 3.081 & 29.479 & 38.874 \\
6 & 0.298 & 0.298 & 0.796 & 1.890 & 2.983 & 59.324 & 34.411 & 40.676 \\
\hline
\end{tabular}

Notes: In addition to restriction I, we discard applicants who do not belong to the top $20 \%$ group selected for procedure A or individuals whose GPA is at least 0.4 grade points better than the threshold needed for admission on the considered rank of list A, or for any university listed on a previous rank (restriction II). $N=8,508$ observations.

Source: Own calculations based on ZVS data on applicants, waves 2005/06 to 2008/09.

Only considering applicants who have good chances of being admitted both in procedures A and $\mathrm{U}$ and who are not prone to strategic behavior, we find the percentage of individuals stating congruent preferences in both procedures increases. $94 \%$ of applicants top-rank the same university in procedures $\mathrm{U}$ and $\mathrm{A}$ (Table 7); the remaining 6\%, however, either do not rank their top-university from procedure $\mathrm{U}$ at all or state it on a lower rank in procedure $\mathrm{A}$. For lower ranks the percentage of deviation again increases. As we discussed earlier, this can be partly due to applicants skipping a university which is recorded as a number of preference reversals further down the list. In order to determine the number of individuals reversing at least one preference, we again exclude double-counting and focus on the first preference reversal within each application. The resulting Table 8 shows that, even after restricting our data set to rule out strategic behavior and carelessness of the applicants, $14 \%$ of all individuals in our data set submit preference lists for procedures A and $U$ that differ in at least one rank. 
Table 8: Preference reversals after restrictions I and II, ruling out strategic incentives and irrelevance (all entries in \%)

\begin{tabular}{c|rrrrrrr}
\hline \hline $\mathrm{U} / \mathrm{A}$ & 2 & 3 & 4 & 5 & 6 & not ranked & total \\
\hline 1 & 3.385 & 0.893 & 0.376 & 0.270 & 0.153 & 1.258 & 6.335 \\
$2^{*}$ & - & 2.092 & 0.646 & 0.317 & 0.176 & 0.870 & 4.101 \\
$3^{*}$ & - & - & 0.835 & 0.458 & 0.118 & 0.482 & 1.893 \\
$4^{*}$ & - & - & - & 0.494 & 0.153 & 0.329 & 0.976 \\
$5^{*}$ & - & - & - & - & 0.282 & 0.306 & 0.588 \\
$6^{*}$ & - & - & - & - & - & 0.235 & 0.235 \\
total & 3.385 & 2.985 & 1.857 & 1.539 & 0.882 & 3.480 & 14.128 \\
\hline \hline
\end{tabular}

Notes: See Table 7

RESULT 7: A significant portion of individuals reveals a preference for randomization when applying for a seat at university that can neither by explained by the characteristics of the application procedure nor by irrelevance of one of the lists for the applicants. In a conservative estimate, we find that $14 \%$ of applicants submit preference lists that differ in at least one rank across procedures.

\subsubsection{Consequences arising from preference reversals}

As our final set of results, we report the frequencies with which applicants who reverse their preferences between lists are matched unnecessarily to suboptimal universities. For this exercise, we restrict attention to applicants who are still in the data set after both restriction I and restriction II - i.e., for whom no rational reasons to manipulate list A exist and for whom both rank-order lists are relevant - and ask the following question: would the applicants who showed preference reversals have been matched to a better university if they had reported their list for procedure $\mathrm{U}$ in both cases? (Here, as before, we assume that applicants report their true preferences on the rank-order list submitted for procedure U, thus "better" refers to the preference recorded on this list.) For the first three of the seven waves of applications, we have the necessary data available to answer the question. Of the $N=5,170$ applications that survive restriction I and restriction II and that appear in these three waves, 650 (13\%) show preference reversals between the two lists, which is comparable to the other waves in 
the data set. Table 9 shows in column (1) what preference rank (on the list for procedure U) was realized for these applicants, and in column (2) what they could have gotten had they simply reported their preference lists for procedure U also for procedure A.

Table 9: Matching outcomes (all entries in \%)

\begin{tabular}{lrrr}
\hline \hline Rank & $(1)$ & $(2)$ & $(3)$ \\
\hline 1 & 68.6 & 85.8 & 88.0 \\
2 & 14.3 & 4.6 & 6.3 \\
3 & 8.3 & 2.9 & 1.7 \\
4 & 2.6 & 2.0 & 1.2 \\
5 & 2.0 & 1.1 & 0.8 \\
6 & 2.5 & 1.2 & 0.4 \\
Other & 1.7 & 2.3 & 1.4 \\
Sum & 100.0 & 100.0 & 100.0 \\
& $N=650$ & $N=650$ & $N=4,520$ \\
\hline
\end{tabular}

Notes: Matching outcomes of $N=5,170$ applicants in three waves. All entries in percent. (1) contains actual matches of all applicants who submitted lists containing preference reversals between A and U. (2) contains hypothetical matches under the counterfactual assumption that the applicants submitted the list for $\mathrm{U}$ under both procedures. (3) contains the actual matches of all applicants who submitted consistent lists.

Source: Own calculations based on ZVS data on applicants, waves 2005/06 to 2006/07.

The comparison of columns (1) and (2) shows that preference reversals substantially harm the individuals who commit them. While only $68.6 \%$ of applicants in this group actually obtain their most preferred university seat, $85.8 \%$ could have obtained their first choice under the counterfactual that they simply report their U-list also under A. The difference is $17.2 \%$ points or a total of 112 applicants in this restricted sample 15 Column (3) shows that the remaining set of 4,520 applicants who submitted consistent lists under A and U had an actual success rate that is very close $(88.0 \%)$ to the counterfactual results of column (2). This indicates that the applicants who reversed their preferences on average faced a similar strategic problem as did those who did not reverse their preferences.

Separate calculations show that the inefficiency becomes even stronger if we further restrict the sample to the 190 applicants (again applying restrictions I and II in the three relevant waves) who simply flip their top two choices between the lists A and U. Of them, only 53.7\%

\footnotetext{
${ }^{15}$ The inefficiencies are even stronger if we consider an alternative counterfactual strategy where the applicants use their preference list for procedure $U$ but truncate it optimally. Instead of $85.8 \%$ as reported in column (2) of Table 9, a total of $92.6 \%$ of the same 650 applicants could have obtained their top choice.
} 
actually obtain their first-listed choice, whereas $82.1 \%$ of them could have obtained their top choice under the counterfactual strategy of sticking to the list for procedure $\mathrm{U}$ and submitting it to both procedures.

RESULT 8: Preference reversals have allocative consequences. Considering only applicants for whom both lists are relevant but who have no rational incentives to submit lists with preference reversals, $17 \%$ could have been allocated to their top-ranked university seat but receive a different seat as a consequence of their reversal. Applicants showing preference reversals have a significantly lower frequency of obtaining their top choice compared to other applicants, but would have an almost equally high chance if they were to submit consistent preferences.

\section{Conclusions}

The paper documents a revealed preference for randomization in a number of different contexts. First, across several experiments we observe that a significant fraction of players choose to randomize between several options. Subjects have to take "difficult" choices in the sense that there is no option that clearly dominates the other(s). Nevertheless, many of the observed choices to randomize appear to have been made deliberately. The observed desire for randomization is robust to variations of the framing: it can be observed when the experimental participants have to make several choices and only one of them becomes relevant with a certain probability, but also when there is the possibility to choose a lottery directly. These direct or indirect randomization choices violate stochastic dominance. Therefore they cannot be justified by any risk attitudes under standard models of choice under uncertainty. Second, in a large dataset on university applications in Germany, a pervasive pattern of reversals of preferences between rank-order lists can be observed, which also induces a suboptimal randomization. In the university-application dataset, significant economic consequences from the 
preference reversals appear.

We have identified a procedural preference that to our knowledge has not been studied yet empirically, apart from studies on procedural fairness. The observed preference for randomization can be interpreted as driven by the desire not to decide oneself, but to delegate the choice to a random process. This can be due to the fear of regret from taking the wrong decision. The motives to avoid a firm choice may well be different between the different data contexts, as it seems natural that different kinds of potential regrets play a role when deciding between universities versus when deciding between vouchers. The experiments clearly cannot tell a complete story for why university applicants leave an important decision to chance unnecessarily. But they can add to the picture by providing evidence that preference reversals and their implied violations of stochastic dominance are sometimes chosen deliberately and not by accident. 


\section{References}

Berg, Joyce E., Dickhaut, John W. and Thomas A. Rietz. 2010. Preference Reversals: The Impact of Truth-Revealing Monetary Incentives. Games and Economic Behavior, 68: 443-468.

Bohra, Abhinash. 2010. Other-Regarding Preferences and Procedural Concerns. Mimeo.

Bolton, Gary E., Brandts, Jordi and Axel Ockenfels. 2005. Fair Procedures: Evidence from Games Involving Lotteries. Economic Journal, 115: 1054-1076.

Braun, Sebastian and Nadja Dwenger: 2009, Success in the University Admission Process in Germany: Regional Provenance Matters?, Higher Education: The International Journal of Higher Education and Educational Planning, 58: 71-80.

Braun, Sebastian, Dwenger, Nadja, Kübler, Dorothea and Alexander Westkamp: 2012, Implementing Quotas in Matching Markets: An Experimental Analysis, Social Science Research Center Berlin (WZB), Discussion Paper No. SP II 2012-201.

Braun, Sebastian, Dwenger, Nadja, and Dorothea Kübler: 2010, Telling the Truth May Not Pay Off: An Empirical Study of Centralized University Admissions in Germany, The B.E. Journal of Economic Analysis 83 Policy (Advances) 10: Article 22.

Camerer, Colin F. and Teck-Hua Ho. 1994. Violations of the Betweenness Axiom and Nonlinearity in Probability. Journal of Risk and Uncertainty, 8: 167-196.

Chen, Yuh-Jia and James E. Corter. 2006. When Mixed Options are Preferred in MultipleTrial Decisions. Journal of Behavioral Decision Making, 19: 17-42.

Gale, David and Lloyd Shapley. 1962. College Admissions and the Stability of Marriage. American Mathematical Monthly, 69: 9-15.

Kahneman, Daniel, Knetsch, Jack L. and Richard Thaler. 1986. Fairness as a Constraint on Profit Seeking: Entitlements in the Market. American Economic Review, 76: 728-741.

Krawczyk, Michal and Fabrice Le Lec. 2008. Give Me a Chance! An Experiment in Social Decision Under Risk., Experimental Economics 13: 500-511 .

Loomes, Graham and Robert Sudgen. 1982. Regret Theory: An Alternative Theory of Rational Choice Under Uncertainty. Economic Journal, 92: 805-824.

Machina, Mark. 1989. Dynamic Consistency and Non-Expected Utility Models of Choice Under Uncertainty. Journal of Economic Literature, 27: 1622-1668.

Read, Daniel and George Loewenstein. 1995. Diversification Bias: Explaining the Discrepancy in Variety Seeking between Combined and Separated Choices. Journal of Experimental Psychology: Applied, 1: 34-49.

Roth, Alvin E. 1982. The Economics of Matching: Stability and Incentives. Mathematics of Operations Research, 7: 617-628. 
Roth, Alvin E. 2008. Deferred Acceptance Algorithms: History, Theory, Practice, and Open Questions. International Journal of Game Theory, 36: 537-569.

Roth, Alvin E. and Elliott Peranson. 1999. The Redesign of the Matching Market for American Physicians: Some Engineering Aspects of Economic Design. American Economic Review, 89: $748-780$.

Roth, Alvin E. and Uriel G. Rothblum. 1999. Truncation Strategies in Matching Markets-in search of Advice for Participants. Econometrica, 67: 21-43.

Roth, Alvin E. and John H. Vande Vate. 1991. Incentives in Two-Sided Matching with Random Stable Mechanisms. Economic Theory, 1: 31-44.

Rubinstein, Ariel. 2002. Irrational Diversification in Multiple Decision Problems. European Economic Review, 46: 1369-78.

Sen, Amartya. 1997. Maximimization and the Act of Choice. Econometrica, 65: 745-779. 


\section{A Appendix}

\section{A1 The Boston algorithm used in procedure A}

The Boston algorithm applied in procedure A can be described as follows:

Step 1: Only the first preferences of the applicants are considered. For each university, admit the selected applicants who have ranked it as their first choice, until there are no seats left or until all candidates ranking this university as their first choice have been admitted. If there are more candidates giving priority to a university than can be admitted, those applicants with the best grades in the Abitur are admitted. Social criteria and (subordinately) lotteries are used to break ties.

Step $k$ : Only the $k^{t h}$ preference of the still unassigned applicants is considered. For each university with available seats, admit the selected applicants who have ranked it as their $k^{\text {th }}$ choice, until there are no seats left or until all candidates ranking the university as their $k^{\text {th }}$ choice have been admitted. If there are more candidates giving the rank $k$ to a university than can be admitted, those applicants with the best average final grade from school are admitted. Social criteria and (subordinately) lotteries are used to break ties.

The algorithm stops after step $k \leq 6$ when every selected applicant is assigned or when all 6 preferences have been considered.

\section{A2 The Gale-Shapley algorithm used in procedure $\mathrm{U}$}

The Gale-Shapley algorithm applied in procedure U works as follows:

Step 1: Each university $i$ with capacity $n_{i}$ offers a seat to the $n_{i}$ applicants it ranks highest. Each applicant tentatively accepts the offer from the university she ranks highest and rejects all remaining offers.

Step $k$ : Each university that was rejected at step $k-1$ by $x$ applicants proposes to its most preferred next choices, with the number of new offers $(x)$ being equal to the number of rejections in the previous round. Each applicant considers the university it has been holding an offer from together with her new offers and tentatively accepts the university she ranks highest and rejects all others.

The algorithm terminates when no proposal by a university is rejected. Each university and applicant is assigned according to the last tentative assignment. If for a certain seat a university is rejected by all applicants to which it has made an offer and there is no applicant left on its preference list, this seat remains unfilled. 


\section{SFB 649 Discussion Paper Series 2013}

For a complete list of Discussion Papers published by the SFB 649, please visit http://sfb649. wiwi. hu-berlin.de.

001 "Functional Data Analysis of Generalized Quantile Regressions" by Mengmeng Guo, Lhan Zhou, Jianhua Z. Huang and Wolfgang Karl Härdle, January 2013.

002 "Statistical properties and stability of ratings in a subset of US firms" by Alexander B. Matthies, January 2013.

003 "Empirical Research on Corporate Credit-Ratings: A Literature Review" by Alexander B. Matthies, January 2013.

004 "Preference for Randomization: Empirical and Experimental Evidence" by Nadja Dwenger, Dorothea Kübler and Georg Weizsäcker, January 2013. 